\title{
SCIENTIFIC CONSIDERATIONS REGARDING THE RESEARCH METHODOLOGY IN CRANIOSACRAL THERAPY FOR CERVICAL PAIN
}

\author{
Anca Maria OLTEAN ${ }^{1 *}$, Marius STOICA ${ }^{1}$, Vasile MARCU ${ }^{1}$, Adina DREVE ${ }^{1}$, \\ Magdalena BUGHIRICÄ ${ }^{1}$ \\ ${ }^{1}$ National University of Physical Education and Sport, Faculty of Physical Education and Sport, Bucharest, \\ Romania \\ *Corresponding author: ancamariaoltean@yahoo.com
}

https://doi.org/10.35189/dpeskj.2021.60.2.6

\begin{abstract}
This paper presents aspects regarding the approach to Craniosacral therapy for people suffering from cervical pain or neck pain. Craniosacral therapy is a gentle manual method of assessing and treating the functioning of the Craniosacral system, which consists of membranes and cerebrospinal fluid that surrounds and protects the brain and spinal cord. The Craniosacral system is closely related to the whole body: any disorder or imbalance can affect organs, muscles and bones as well as the nervous, circulatory or endocrine systems. Through Craniosacral therapy, these imbalances can be felt/palpated and rectified by very gentle manipulations on the skull or sacrum bone. The beneficial influence of Craniosacral therapy can be clearly determined especially in the case of disorders of the central nervous system. Craniosacral therapy improves the general condition of the body, reduces stress and tension and enhances vital functions, thus increasing the quality of life of patients. The main objectives of this paper refer to the optimisation of Craniosacral therapy and the holistic approach to human personality. The current research is based on elements of novelty that have not been validated so far in the Romanian literature: it detects the possibilities of exploiting the improvement of the treatment applied to people with cervical pain.
\end{abstract}

Keywords: Craniosacral therapy, quality of life, cervical pain.

\section{Introduction}

The father of Osteopathy is the physician Andrew Tylor Still (1828-1917), who was born in America, Virginia. On June 22, 1874, he decided to lay the foundations of Osteopathy following several good results in treating diseases that had no cure at that time. Between 1878 and 1885 , he practised Osteopathy and gradually gave up prescribing drugs. Overwhelmed by the large number of people requesting his services due to the medical results obtained, he founded the first school for osteopathic medicine, the American School of Osteopathy, in 1892. Osteopathy is a holistic system of diagnosis and treatment that distinctly addresses the structural and biomechanical problems of the human body in close interdependence with its psycho-emotional status.

The term "osteopathy" comes from the Greek "ostheon" = bone and "pathos" = condition, thus designating diseases that have a structural origin. Osteopathy uses a whole arsenal of methods and techniques of manual medicine (joint, visceral, cranial osteopathy) adapted for all ages and types of tissues (fascial, muscle, ligament, bone, visceral ones), with the ultimate goal of restoring the structural balance of the body.

Studying the interdependence of all parts of the body, Dr Still has found that acting on one area of the body influences the whole body. If imbalances in the functioning of tissues are 
treated in due time, then the disease process caused by their malfunction can be changed or even reversed. He states that Osteopathy is "anatomy, anatomy and anatomy" and that the limit of Osteopathy is only the therapist and their ability to understand what happens in a dysfunction. In 1892, Dr Still said: "To find health should be the object of the doctor. Anyone can find the disease". (Upledger, 2013, pp. 28-30)

The four principles of Osteopathy are (Liem \& Tsolodimos, 2016):

1. The principle of globality of the body - the human body is a unitary whole made up of body, mind and soul;

2. The principle of homeostasis - the body is able to self-regulate, heal itself and stay healthy;

3. The principle of interdependence between structure and function - the structure and functions of the body influence each other;

4. The principle that life is movement - any osteopathic treatment follows the three previous principles. The unity of the body, its self-healing ability and the interrelationship between structure and function are taken into account.

The foundations of the Craniosacral therapeutic technique were laid between 1930 and 1940 by Dr William Garner Sutherland (1873-1954), one of Dr Still's students. He has medically proven that the cerebrospinal fluid (irrigating the brain and spinal cord) has a rhythmic pulse, which consists of flexion (expansion of the fluid) and extension (withdrawal of the fluid). This pulse can be felt/palpated in any bone or tissue because the fluid irrigates the entire body and penetrates everywhere. The pulse (6-12 cycles per minute) works harmoniously and undisturbed only when the bones of the skull have some elasticity and can follow the filling and emptying phases of the cerebrospinal fluid. He also pointed out the importance of cranial bone motion. (Upledger et al., 2008)

In 1970, the osteopathic doctor John E. Upledger allopathically proves the existence of the Craniosacral rhythm; thus, he demonstrates that the spinal meningeal membrane has a rhythmic pulse, and this rhythm plays an important role in emotional balance, a phenomenon that occurs instantly when restoring the Craniosacral rhythm. Dr Upledger supports this with scientific studies conducted between 1975 and 1983 at the Michigan State University where he worked as a clinical researcher and Professor of Biomechanics. He wrote many books and articles and recorded DVDs on Craniosacral therapy, receiving several awards as an innovator and mentor in discovering new therapies. His involvement in the development of Craniosacral therapy has been recognised internationally. He is a co-founder of the Upledger Institute, now called the Upledger Institute International, the largest Craniosacral therapy school that was established in 1985 and has branches in 59 countries around the world.

Craniosacral therapy techniques are taught to health professionals, osteopaths, psychiatrists, doctors, dentists, chiropractors, physiotherapists, masseurs and nurses within the Upledger Institute programme. Craniosacral therapy is a gentle manual non-invasive method of assessing and treating the functioning of the Craniosacral system, which consists of membranes and cerebrospinal fluid that surrounds and protects the brain and spinal cord. Commonly using a gentle touch of 5 grams, the therapist releases constraints from the Craniosacral system in order to improve the functioning of the central nervous system, which leads to somatic and mental relaxation. The Craniosacral system is closely related to the 
whole body: any disorder or imbalance can affect organs, muscles and bones as well as the nervous, circulatory or endocrine systems (Upledger, 2013).

Through Craniosacral therapy, these imbalances can be felt/palpated and rectified by very gentle manipulations on the skull or sacrum bone. The beneficial influence of Craniosacral therapy can be clearly determined especially in the case of disorders of the central nervous system. Craniosacral therapy improves the general condition of the body, reduces stress and tension and enhances vital functions. The belief behind Craniosacral therapy is that the human body has the ability to heal itself. In addition to reducing pain, stress and tension in the body, the Craniosacral step can increase a person's understanding of their inner energy and their body's self-healing potential. Awareness of one's own body, stress level and inner tension is very important for disease prevention. By paying attention to our body and feeling the changes that take place inside it, we can take timely measures to prevent disease, prevention being a very important aspect that has been much emphasised in recent times. Strong tension or overuse of the fibrous connective tissue affects the fascia and muscles in terms of mobility and position of the surrounding bones and joints. The consequences are recurrent pain, a tendency to muscle tension and even injuries. Craniosacral therapy acts directly on the connective tissue and bones. The inner tension disappears and the whole body aligns harmoniously. (Liem \& Tsolodimos, 2016)

One of the preliminary requirements for working with the Craniosacral system is the discovery (made in this century) that the various bones of the skull, which are connected to each other by sutures, are mobile. This is generated by the brain flow and the cerebrospinal fluid motion. In the skull, the cerebrospinal fluid and dura mater are influenced by the mild pressures used in therapy. Perceiving the interaction of different bones and membranes of the skull, which are felt down to the sacrum and influence each other - this is the purpose of Craniosacral therapy.

The functional unit of this form of therapy has been described since the Craniosacral system was given this name (Mackinnon, 2014):

- it involves the unity between the skull and sacrum, including the connecting structures of the spine and dura mater, as well as the paravertebral muscles and the nervous system;

- it also includes the cerebrospinal fluid and all structures that correlate with its production, transport and resorption;

- it is closely related to the nervous, circulatory, lymphatic, endocrine, respiratory and musculoskeletal systems;

- it is the centre of our energy system, which provides the body with energy;

- disorders in this system have an effect on the whole body, but Craniosacral balancing enables to restore disorders and problems throughout the body;

- the Craniosacral system is well assessed and implemented as a tool for accurate diagnosis and treatment.

In addition to cardiovascular and respiratory rhythms, there is a third very important rhythm in the body, namely the Craniosacral rhythm or Craniosacral breathing. The Craniosacral rhythm is a deep rhythm, which is very close to nature and is perceptible from the moment of egg fertilisation up to 30 minutes after death. The Craniosacral rhythm has a 
frequency of 6-12 cycles per minute. This rhythm can be felt throughout the body and can differ, as well as frequency, in different parts of the body. It consists of two phases:

- the first phase involves pressure that leads to the expansion of the skull;

- in the second phase, the Craniosacral system gets relaxed, the pressure is reduced, and the skull returns to its original shape.

These phases are repeated 6-12 times per minute. Because the Craniosacral system is a basic vital system, it is also called the "primary respiratory mechanism" or "basic breathing" of the body. This "basic breathing" moves the bones of the skull almost imperceptibly through their sutures. This rhythm is felt not only in the area between the skull and the sacrum bone but also in the whole body like a closing and opening wavy movement. Where this rhythm is not felt, very fine correction impulses can be applied using certain manual techniques. Each disease and each energy blockage cause a specific deviant pattern from the specific rhythm of "basic breathing". The role of Craniosacral therapy is to restore this rhythm to normal parameters.

Complementing the body's natural healing system, Craniosacral therapy is more and more used as a means of prevention due to its ability to increase disease resistance and its effectiveness in a wide range of medical problems associated with pain and dysfunction such as those presented in Figure 1 (Upledger, 2018):

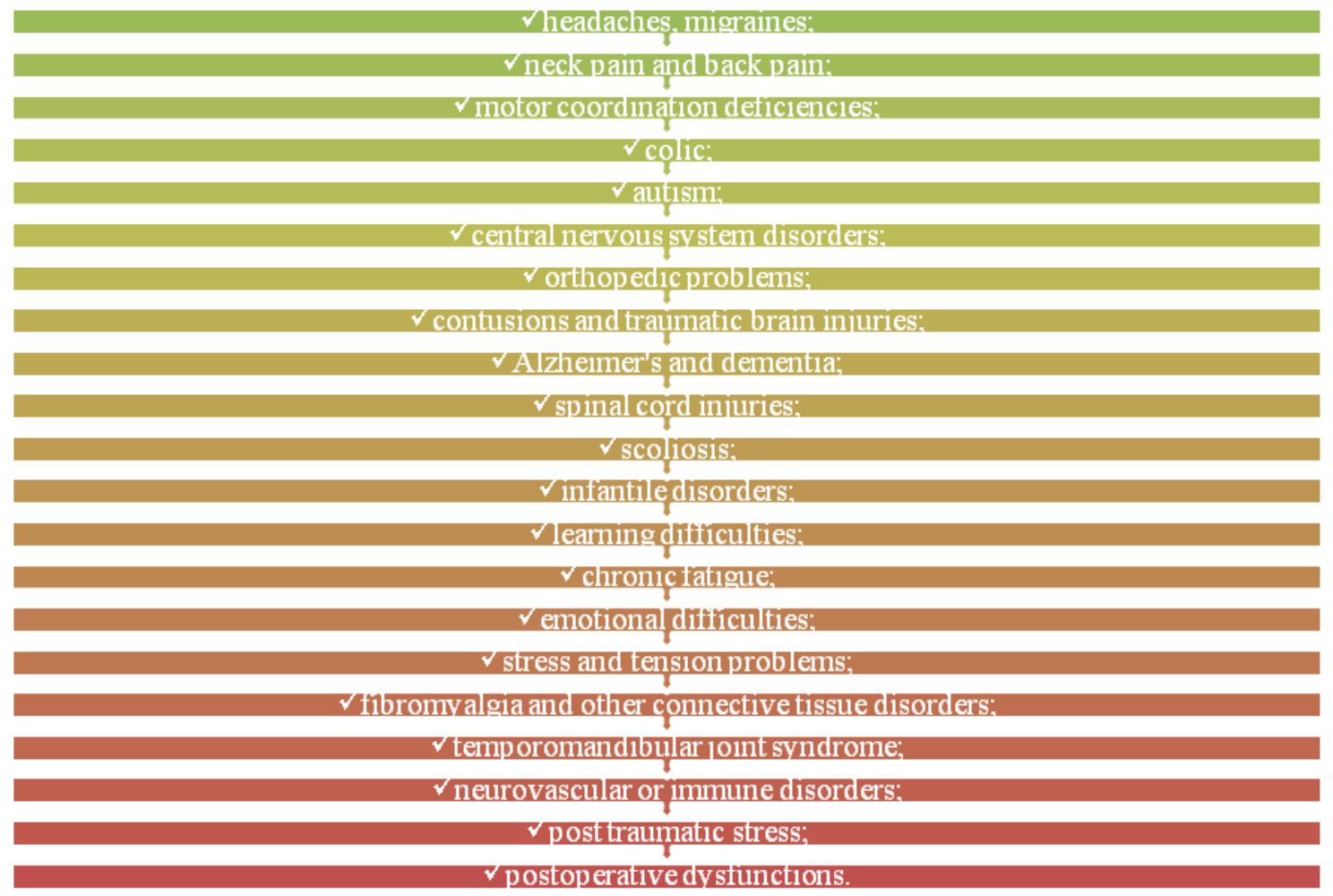

Figure 1. Medical problems for which Craniosacral therapy can be used

Craniosacral therapy can also be used in animals for various minor injuries or nervousness, which deepens and strengthens the relationship with the pet. 
A Craniosacral therapy session lasts between 30 minutes and 1 hour or even 1 hour and a half. The patient lies supine on the treatment table while being fully dressed in light clothes. The therapist's palms will be placed on the legs, along the body, on the chest or head of the patient and will support their head, spine or sacrum to eliminate tensions accumulated in the body. The environment in which Craniosacral therapy sessions take place should be comfortable, quiet, pleasant for both the patient and the therapist in order to induce a state of calm, relaxation, thus facilitating the relief of pain, symptoms associated with anxiety, panic attacks or depression. The manoeuvres performed by the therapist are light and gentle, giving the patient a feeling of well-being and peace. The patient can focus on breathing during treatment to achieve a state of relaxation by increasing the activity of the parasympathetic nervous system. Another phenomenon called SomatoEmotional Release may occur (although not necessarily during each treatment) as a result of recalling traumatic and/or emotional moments. This is a normal phenomenon that helps the body reverse the dysfunction and restore the optimal level of mobility, to free the mind and body from the residual effects of trauma. (Upledger, 2013)

Specific procedures for Craniosacral therapy used by therapists include compressiondecompression of the temporomandibular joint, decompression of the mandibular fascia, facial bone strain, compression-decompression of the sphenobasilar joint, parietal lifting, frontal lifting, diaphragm belt release, scapular girdle decompression of the sacrum, compression-decompression of the iliosacral joint and iliac crests. Still points are quiet intervals between manipulation techniques when the Craniosacral rhythm stops, which happens every 3 to 4 minutes and lasts for a minute, during which the patient rests quietly. Craniosacral therapy is addressed to both people with health problems and healthy people who want to maintain their normal tone in order to cope with daily stress more easily. It is suitable for all age groups, from the newborn to the third age but also pre-postpartum women, to reduce the risk of worsening subsequent symptoms; however, Craniosacral therapy is not recommended for people whose intracranial pressure can cause instability as in the case of the following conditions: acute aneurysm, cerebral haemorrhage, brain tumours or oedema, epilepsy, recent spinal cord injury, severe bleeding disorders. (Upledger et al., 2008)

The study by Hazelbaker (2020) confirms that this therapy, together with osteopathic therapy, has positive effects on breastfeeding through the process of rectifying the patterns of strains that have a structural function in children.

The results obtained by Haller et al. (2019) showed that Craniosacral therapy applied after 6 months had greater post-intervention effects on pain intensity compared to conventional therapies. Craniosacral therapy also appears to be effective and safe for childhood colic by reducing the number of hours of crying, the severity of colic and the total increase in sleep hours (Castejon-Castejon et al., 2019).

Researchers in Iceland studied the effect of Craniosacral therapy on patients with headaches/migraines. Participants with a diagnosis of migraine headache having two or more episodes of pain in the month prior to the study were recruited. Participants aged 18 to 50 years were randomly assigned to two groups (A or B) and could continue their daily activities and prescribed medication. No other form of alternative treatment was allowed. Craniosacral therapy was applied, and the result showed a significant decrease in pain intensity immediately after the therapy and during the subsequent 4 weeks. Although the study was 
limited by the small number of participants and the lack of a control group, it was selected for review because it was a good pilot project. (King, 2017)

Castro-Sanchez et al. (2016) conducted a randomised controlled trial with 64 patients suffering from chronic nonspecific low back pain, who had been included in a recovery programme. For this study, two groups were created - an experimental and a control one. Patients assigned to the experimental group performed 10 sessions of Craniosacral therapy, while those in the control group performed 10 sessions of classic massage. The study concluded that patients receiving Craniosacral therapy had a greater improvement in pain intensity compared to those receiving classic massage.

Haller et al. (2016) conducted a study on patients receiving 8 weekly Craniosacral therapy sessions or another treatment and reported the effects of Craniosacral therapy on pain management. The results were assessed before and after 8 weeks of treatment and again after 20 weeks. The main outcome was a reduction in pain intensity on a visual analogue scale at week 8, while "secondary outcomes included pain on movement, pressure pain sensitivity, functional disability, quality of life related to health, well-being, anxiety, depression, stress perception, pain acceptance, body awareness, patients' global impression of improvement and safety" (Haller et al. 2016, p. 441). Patients who received Craniosacral therapy reported significant and clinically relevant effects on pain intensity at week 8 . Significant differences between groups at week 20 were also found for pain on movement, functional disability, physical quality of life, anxiety and patients' global improvement. Body awareness and pressure pain sensitivity were significantly improved at week 8 . No serious adverse effects were reported. Therefore, Craniosacral therapy is effective and safe in reducing neck pain intensity and improving quality of life and functional disability for up to 3 months posttreatment.

In a multicentre, randomised controlled study conducted in hospitals and other care centres in Sweden, Elden et al. (2013) draw attention to the effects of Craniosacral therapy as adjunct to medical treatment for pelvic pain in pregnant women. The women were divided into two groups; those in the control group received standard treatment, and the experimental group received standard treatment and Craniosacral therapy. It was found that morning pain intensity was lower in women who had received Craniosacral therapy, but there were no effects on evening pain. Given that treatment effects were small, further research is needed.

Arnadottir and Sigurdardottir (2013) present in their study the effect of Craniosacral therapy on reducing migraine symptoms in patients aged 20 to 50 years (the participants received 6 sessions of Craniosacral therapy over 4 weeks). After one month, there was a significant decrease in migraines for those who had benefited from Craniosacral therapy.

Jakel and Von Hauenschild (2012) make a systematic analysis that presents clear scientific evidence on the beneficial role of Craniosacral therapy for patients. The conclusion of the study also demonstrates a lack of research on Craniosacral therapy in multiple pathologies. Indeed, this therapy has valuable potential and can bring a plus in clinical decision-making, but additional studies are needed.

The research by Mataran-Panarrocha et al. (2011) reports the effects of Craniosacral therapy on depression, anxiety and quality of life in patients with painful symptoms of fibromyalgia. The clinical study was conducted on 84 patients diagnosed with this pathology. The therapy was applied for 25 weeks, and the results were determined at 10 weeks, 6 months 
and 1 year after treatment. Thus, anxiety and pain were reduced, while sleep quality and quality of life were improved in the intervention group compared to the placebo group. In conclusion, this therapy applied to patients with fibromyalgia contributes to reducing their anxiety and improving their quality of life.

A descriptive study by Harrison and Page (2011) demonstrates the effectiveness of Craniosacral therapy in general. The treatment was applied to 157 people with various medical problems such as headaches, migraine, sore throat, back pain, anxiety and depression. The results showed that $74 \%$ of patients reported a significant improvement in their health problem, $67 \%$ reported an improvement in quality of life, a reduction in pain and chronic stress, and $70 \%$ reported a decrease or even discontinuation in their medication.

The aforementioned scientific studies prove the effectiveness of using Craniosacral therapy in cervical pain.

\section{Topic Addressed}

Due to the fact that many young people, women, adults, seniors show up at the office with various and uncomfortable back pain symptoms in general or cervical pain, we believe that an updated thorough research is needed on this topic. Cervical pain is the most common type of pain after headache and low back pain. It can be caused by a specific activity or trauma to the neck or by another medical condition. Most cases of neck pain are caused by activities that involve repeated and prolonged movements of the neck muscles, ligaments, tendons, bones and joints. This can lead to stretching (excessive tension or muscle overload), sprain (ligament injury) and spasm of the cervical muscles or inflammation of the cervical joints.

Injuries that occur in everyday life either accidentally or at work, on the sports field, in the street, in traffic, can cause (at the very best) a sprain or dislocation of the spine but also fractures or damage to the spinal cord, which may result in paraplegia or tetraparesis.

For over 4 years, one of the co-authors of this study had the chance to work in the specialised field of physiotherapy in Austria, in several clinics or outpatient services, and had the opportunity to apply the methods taught at the University of Oradea with satisfactory results. Having access to more information and specialised courses, several courses were taken in Osteopathy, Craniosacral therapy, various manual therapies (Trigger Point, passive stretching), techniques for applying kinesiological bands, functional training methods, Feldenkreis, Smovey Training, thus improving patient approach techniques and therapeutic methods.

The new techniques learned abroad were applied in Romania to several people with spinal problems of different ages and professions. Many of them complained of pain in the cervical spine. Using the knowledge acquired in Osteopathy and Craniosacral therapy courses, a significant improvement was found in patient outcomes: reduced pain, greater joint mobility, improved sleep quality, increased comfort in daily activities and improved quality of life.

To assess patients, both subjective and objective techniques were used. Pain assessment: location, irradiation - when pain occurs at rest or while moving, intensity - measured on a numerical scale of 1 (no pain) to 10 (maximum pain), interference with daily activity, mood, relationships with colleagues, friends and family, sleep quality, pain variation over time. 
- Assessment of spinal mobility - the mobility and suppleness of the cervical spine is assessed by measuring the distance between:

- ear (tragus) and shoulder (acromion) for right-left lateral tilt movements, the normal value being 0 ; chin (chin) and shoulder (acromion) for right-left rotational movements;

- chin (chin) and chest (sternum) for flexion-extension movements, the normal value being 0 in flexion;

- occiput and wall; the distance is measured in orthostatism with the back, buttocks and heels pressed against the wall, the normal value being 0 .

- Muscle strength assessment: manual muscle testing - remains the most used method for assessing muscle strength by a rating system equivalent to the one proposed by Lovett and Martin (1916) on a scale from F0 = the muscle does not achieve any obvious contraction to F5 $($ normal $)=$ the muscle is able to mobilise the segment on the entire range of motion against maximum resistance applied on the segment to be mobilised as distally as possible.

The World Health Organization (2012) defines quality of life as "individuals' perceptions of their position in life in the context of the culture and value systems in which they live and in relation to their goals, expectations, standards and concerns" (p. 11).

According to Jenkinson (2020), quality of life is the degree to which an individual is healthy, comfortable and able to participate in or enjoy life events. These are very important things to consider, and Craniosacral therapy plays a major role in this regard.

Depending on personal functional assessments, general and operational objectives and personalised Craniosacral therapy programmes were established. It is worth mentioning that Craniosacral therapy sessions took place in a melo-therapeutic setting specific to each patient, according to their moods and preferences on the day of treatment. Agitated or nervous patients were given soothing, calming musical compositions, and those depressed or demoralised benefited from stimulating musical pieces (for example, Mozart for reassurance, Beethoven for stimulation). The aromatherapy lamp with essential oils was also used, depending on each patient's preferences and needs on the day of treatment (for example, lavender, chamomile or sage essential oil for relaxation and soothing, peppermint, lemon or ylang-ylang essential oil for stimulation and invigoration).

During a Craniosacral therapy session, the therapist aims to feel the Craniosacral rhythm throughout the patient's body and thus find where it is not the same, where there are blockages so as to provide gentle intervention to normalise it and help the body to unblock and move freely. The therapist also follows the moments of Still points that are quiet intervals between the manipulation techniques where the Craniosacral rhythm stops, which happens every 3 to 4 minutes and lasts for a minute. At these times, the therapist does not perform any technique but simply lets the patient rest.

The techniques used in Craniosacral therapy are indirect and direct. Indirect techniques compare the amplitude of flexion and extension of the Craniosacral rhythm. The therapist follows the movements of the Craniosacral rhythm that they feel. If the therapist feels at some point that something in the structure of the muscle fascia, tissue or joint will resist the movement but after a few moments gives way, then the amplitude of the movement felt can be increased a little more. Then the movement stops and the tissue structure gets relaxed. The indirect technique is the gentlest method and is always used on the bones of the skull. 
In direct techniques, the process is the same but the therapist tries not to wait for the time of tissue release and extends it to the next level where the relaxation period follows. Specific techniques applied in the treatment of neck pain include:

- Atlas-Axis - this technique removes the atlas bone from the base of the skull and the atlas from axis. The patient lies supine on the therapy bed, and the therapist sits at the patient's head holding it in both palms with fingertips positioned at the junction of the skull with the cervical spine (Figure 2). The weight of the head is used to apply the correct pressure to relax the cervical muscles.

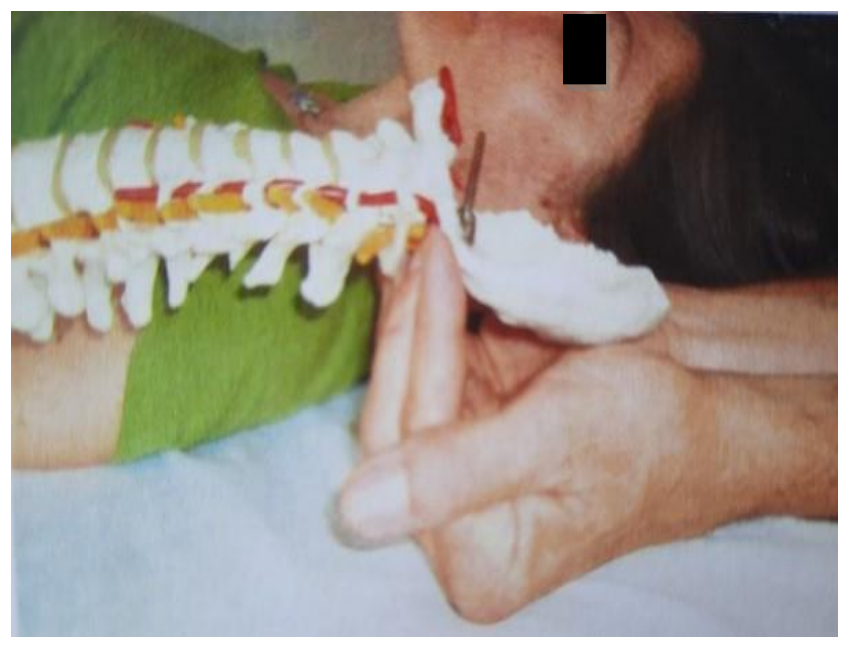

Figure 2. Atlas-Axis decompression

- Occipital bone decompression - the patient lies supine, and the therapist grabs the patient's occipital bone with 2-4 extended fingers of both hands, which slide towards each other until they meet, and then follows the Craniosacral rhythm (Figure 3). This helps a lot to relax.

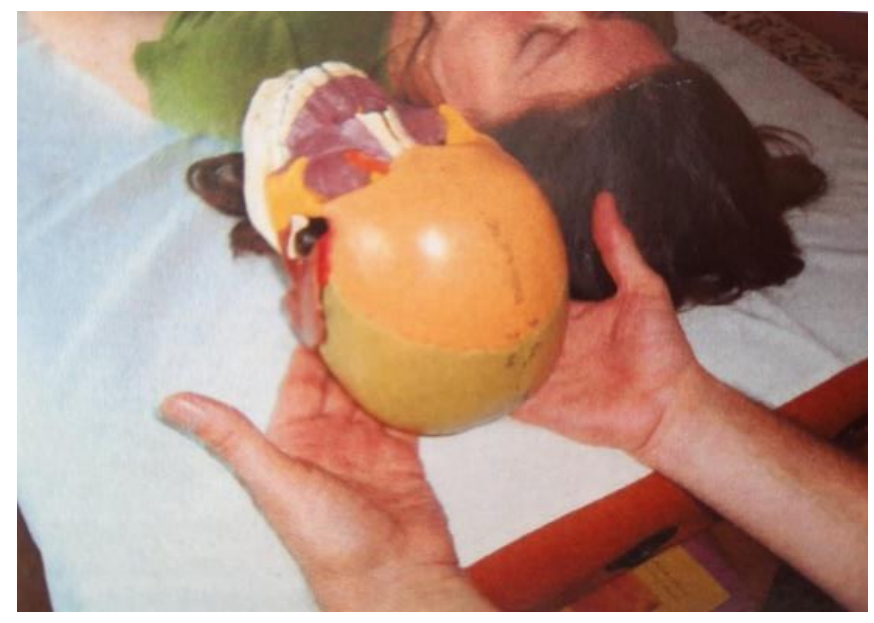

Figure 3. Occipital bone decompression 
- CV4 (cranial ventricle 4) - a slight ventral compression of the occipital bone stimulates the cranial ventricle 4 , thus supporting the exchange of cerebrospinal fluid in the brain and subsequently helps the system to renew itself (Figure 4).

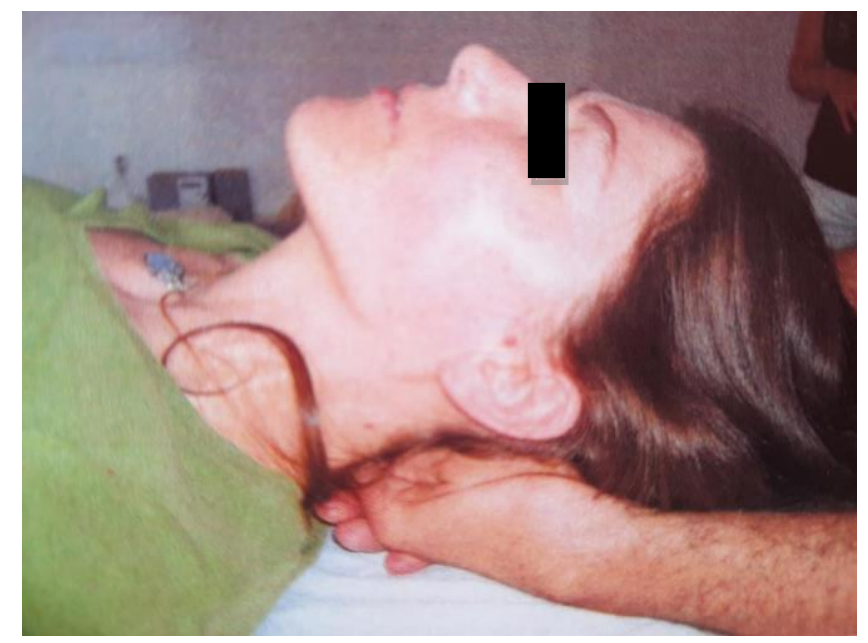

Figure 4. CV 4

- Frontal, sphenoid, parietal and temporal bone decompression - the therapist's fingers are spread out to form a fan-like shape: the thumbs are on the frontal bone, the index fingers are placed on the sphenoid and parietal bones, the middle and ring fingers are on the temporal bones, and the small fingers reach the occipital bone (Figure 5).

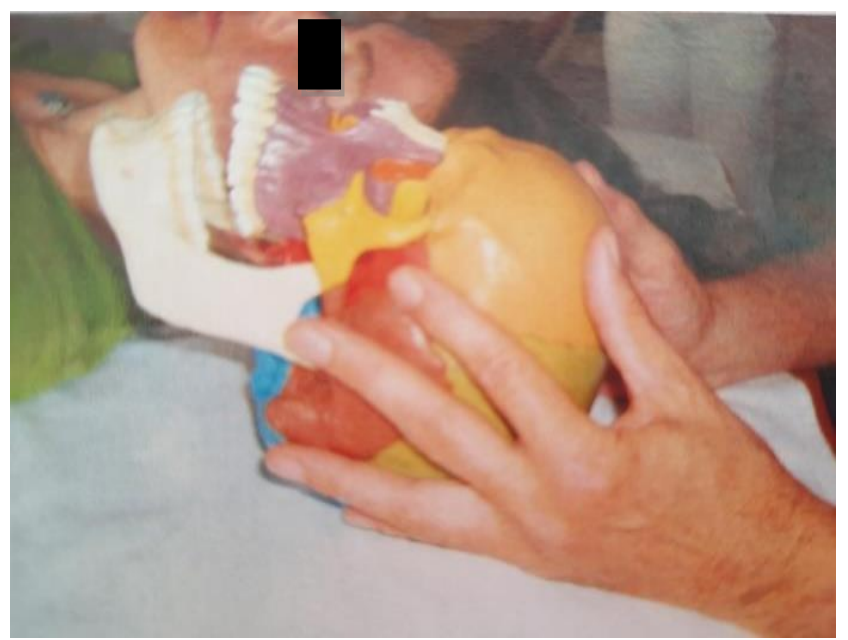

Figure 5. Frontal, sphenoid, parietal and temporal bone decompression

\section{Conclusion}

Compliance with the principles of Craniosacral therapy is essential to achieve the desired outcomes, otherwise failure may occur with possible repercussions for patients. Craniosacral therapy is a gentle manual non-invasive method that allows to feel imbalances in the Craniosacral system and rectify them by very gentle manipulations on the skull or sacrum. 
The main concept underlying Craniosacral therapy is that the human body has the ability to heal itself. In addition to reducing pain, stress and tension in the body, the Craniosacral step can increase a person's understanding of their own inner energy and the body's selfhealing potential. Awareness of one's own body, stress level and inner tension is very important for disease prevention. By paying attention to our body and feeling the changes that take place inside it, we can take timely measures to prevent disease, prevention being a very important aspect that has been much emphasised in recent times.

It should be specified that each disease and each energy blockage cause a specific deviant pattern from the specific rhythm of "basic breathing". The role of Craniosacral therapy is to restore this rhythm to normal parameters. Numerous studies presented in this paper show that Craniosacral therapy has medium-term beneficial effects on various diseases of the nervous system after discontinuation of therapy. These effects can be seen in patients who have systematically undergone treatment over periods between 1 and 6 months. Improving the quality of life of patients is a very important aspect to be taken into account.

The application of Craniosacral therapy according to established principles and rules is essential for obtaining the expected effects. Any mistake made by the therapist when applying it may lead to errors in achieving the final results.

\section{References}

Arnadottir, T. S., \& Sigurdardottir, A. K. (2013). Is Craniosacral therapy effective for migraine? Tested with HIT-6 Questionnaire. Complementary Therapies in Clinical Practice, 19(1), 11-14. https://doi.org/10.1016/j.ctcp.2012.09.003

Castejon-Castejon, M., Murcia-Gonzalez, M. A., Gil, J. L. M., Todri, J., Rancel, M. S., Lena, O., \& Chillon-Martinez, R. (2019). Effectiveness of Craniosacral therapy in the treatment of infantile colic. A randomized controlled trial. Complementary Therapies in Medicine, 47: 102164. https://doi.org/10.1016/j.ctim.2019.07.023

Castro-Sanchez, A. M., Lara-Palomo, I. C., Mataran-Penarrocha, G. A., SaavedraHernandez, M., Perez-Marmol, J. M., \& Aguilar-Ferrandiz, M. E. (2016). Benefits of Craniosacral therapy in patients with chronic low back pain: A randomized controlled trial. Journal of Alternative and Complementary Medicine, 22(8), 650-657. https://doi.org/10.1089/acm.2016.0068

Elden, H., Ostgaard, H. C., Glantz, A., Marciniak, P., Linner, A. C., \& Olsen, M. F. (2013). Effects of Craniosacral therapy as adjunct to standard treatment for pelvic girdle pain in pregnant women: A multicenter, single blind, randomized controlled trial. Acta Obstetricia et Gynecologica Scandinavica, 92(7), 775-782. https://doi.org/10.1111/aogs.12096

Haller, H., Lauche, R., Sundberg, T., Dobos, G., \& Cramer, H. (2019). Craniosacral therapy for chronic pain: A systematic review and meta-analysis of randomized controlled trials. BMC Musculoskeletal Disorders, 21(1): 1. https://doi.org/10.1186/s12891-019-3017-y

Haller, H., Lauche, R., Cramer, H., Rampp, T., Saha, F. J., Ostermann, T., \& Dobos, G. (2016). Craniosacral therapy for the treatment of chronic neck pain: A randomized shamcontrolled trial. Clinical Journal of Pain, 23(5), 441-449.

https://doi.org/10.1097/ajp.0000000000000290

Harrison, R. E., \& Page, J. S. (2011). Multipractitioner Upledger Craniosacral therapy: Descriptive outcome study 2007-2008. The Journal of Alternative and Complementary Medicine, 17(1), 13-17. https://doi.org/10.1089/acm.2009.0644 
Hazelbaker, A. K. (2020). The impact of Craniosacral therapy/cranial osteopathy on breastfeeding. Clinical Lactation, 11(1), 21-27. https://doi.org/10.1891/2158-0782.11.1.21

Jakel, A., \& Von Hauenschild, P. (2012). A systematic review to evaluate the clinical benefits of Craniosacral therapy. Complementary Therapies in Medicine, 20(6), 456-465. https://doi.org/10.1016/j.ctim.2012.07.009

Jenkinson, C. (2020). Quality of life. In Encyclopedia Britannica. https://www.britannica.com/topic/quality-of-life

King, H. H. (2017). Manual Craniosacral therapy may reduce symptoms of migraine headache. Journal of the American Osteopathic Association, 117(1): 59. https://doi.org/10.7556/jaoa.2017.011

Liem, T., \& Tsolodimos, C. (2016). Osteopathie: Gezieltes Lösen von Blockaden [Osteopathy: Targeted release of blockages]. Georg Thieme Verlag KG.

Lovett, R. W., \& Martin, E. G. (1916). Certain aspects of infantile paralysis and a description of a method of muscle testing. JAMA, 66(10), 729-733. https://doi.org/10.1001/JAMA.1916.02580360031009

Mackinnon, K. (2014). Din inima și mâinile mele: Cum să obținem sănătate și echilibru prin terapia craniosacrală [From my hands and heart: Achieving health and balance with Craniosacral therapy]. Hay House.

Mataran-Penarrocha, G. A., Castro-Sanchez, A. M., Garcia, G. C., Moreno-Lorenzo, C., Carreno, T. P., \& Zafra, M. D. O. (2011). Influence of Craniosacral therapy on anxiety, depression and quality of life in patients with fibromyalgia. Evidence-Based Complementary and Alternative Medicine, 2011: 178769. https://doi.org/10.1093/ecam/nep125

Upledger, J. E. (2013). Auf den Inneren Arzt hören Eine Eifürung in die CranioSacrale Tharapie [Listening to the inner doctor: An introduction to Craniosacral therapy]. Irisiana.

Upledger, J. E. (2018). Tu și medicul din tine: Terapia craniosacrală și eliberarea somatoemoțională [Your inner physician and you: Craniosacral therapy and somatoemotional release]. Herald.

Upledger, J. E., Grossinger, R., Ash, D., \& Cohen, D. (2008). Craniosacral therapy: What it is. How it works. North Atlantic Books.

World Health Organization. (2012). WHOQOL: Measuring quality of life. https://www.who.int/tools/whoqol 